AIAA 2002-2874

\title{
ANALYSIS OF STEFAN PROBLEM WITH LEVEL SET METHOD
}

\author{
Wonjoo Roh* and Noboru Kikuchi $\dagger$ \\ University of Michigan, Ann Arbor, Michigan 48109-2125
}

\begin{abstract}
Numerical investigation of Stefan problem is conducted. The method developed is a combination of front tracking method and fixed domain method by finite element method. The fixed entire domain is applied to a melting/solidification problem by finite element method without depending on enthalpy formulation. The moving front is tracked by level set method based on Eulerian approach. The method can deal with several frozen fronts, which develop simultaneously and collapse each other. Numerical result for three dimensional melting problem with multiple melting fronts is presented.
\end{abstract}

\section{Introduction}

Stefan problem is one of moving/free boundary problems, which include shock waves in gas dynamics, crack propagations in solid mechanics, and fluid flow through porous media. The problems should satisfy certain conditions on the moving boundary of which have to be determined as a function of time and space. J. Stefan (1891) interested in the melting of the polar ice cap studied it for the first time. He derived the large latent-heat approximation and extended this solution to include time-dependent surface temperature. In fact the more general result known as Neumann's solution was given by F. Neumann in his lectures in the 1860's.

Very few analytical solutions are available in closed form. Carslaw and Jaeger ${ }^{1}$, Tikhonov ${ }^{2}$, and Crank ${ }^{3}$ reproduced and described the similarity solutions in detail. They are mainly for the one-dimensional cases of an infinite or semi-infinite region with simple initial and boundary conditions and constant thermal properties. There are no close solutions for other important boundary conditions such as constant flux or radiation. The Stefan problem is necessarily nonlinear problem because the jump condition for the unknown interfaces and the governing energy equations are coupled even if all the thermal properties are assumed to be constant. Carslaw and Jaeger ${ }^{1}$ explained the nonlinearity of Stefan problem. Then, apart from the few exact solutions, all problems, especially higher dimensional problems, have to be attacked by numerical methods.

There have been two major trends for solving a moving boundary Stefan problem by numerical computations. One is front-tracking method and the other is fixed-domain method. The former method

\footnotetext{
*Graduate Student, Department of Mechanical Engineering, 3005 EECS, 2350 Hayward Street

$\dagger$ Professor, Department of Mechanical Engineering, 3005 EECS, 2350 Hayward Street
}

includes finite difference method on both a fixed grid and various space grid, body-fitted method, isothermal migration method and finite element method with an adaptive mesh. The latter method includes enthalpy method and variational inequality method. The main difference between those two methods is that the solution field such as temperature is defined all over the analysis domain or not. For example, if the moving front is not detected priori, the governing energy equation cannot be solved in front-tracking method because the solution field is defined only on a confined area (solid/liquid). After transforming the solution fields into a different variable such as enthalpy or freezing index, these variables are defined for all over the domain and the problem can be solved without tracking the moving front explicitly. The interface is given, a posteriori, by the value of solution field.

Crank $^{4}$, Ehrlich ${ }^{5}$, and Koh et at solved it with a fixed grid by finite difference method. They used different approach to calculate the first and second derivatives of the solution field at the moving front. The interpolation scheme used by Crank was least squares method and that of Ehrlich ${ }^{5}$ and Koh et al ${ }^{6}$ was Taylor expansion. An alternative to tracking the moving front is to fix it by a suitable choice of new space coordinates. The new curvilinear coordinates are sensibly referred to as body-fitted coordinates. For one dimensional case, Crank $^{7}$ applied the transformation such as $\xi=x / s(t)$ into finite difference scheme. Lotkin ${ }^{8}$ used unequal intervals in $\xi$ and $t$, and divided differences to obtain an economic improvement in accuracy. The body-fitted method could make use of fixed domain for the analysis; however, it produced the complexities into the transformed governing equations. Kim and Kaviany ${ }^{9}$ tried to remove the complexities through the careful use of a coordinate transformation. The method using an adaptive mesh refines the mesh at each time step so that the phase interface always remains on boundaries between elements. However, this method needs built-in 
remeshing capabilities so that it is computationally expensive. Also, for large geometric changes in moving boundaries, the mesh may become distorted to produce inaccurate solutions so that it is restricted to one dimension or simple interfaces in higher dimensions.

The essential feature of enthalpy method is that the two or more heat equations in different phases can be reformulated by the single equation and the moving interface is given after solving the problem. However, the enthalpy function is discontinuous at the melting / solidifying moving front and the phase boundary cannot be maintained as a sharp front. The reason for it comes from the definition of enthalpy function so that the moving front is spread with a fin ite gap between phase changes. Duvaut introduced special variable, which was referred by Fremond ${ }^{10}$ as the 'freezing index', and this index led to the variational inequality formulation instead of a usual weak form. Yichikawa and Kikuchi ${ }^{11}$ solved one and two phase Stefan problem by using the freezing index transformation. However, accurate tracking of moving fronts may not be accomplished because the Stefan condition is already included into the governing equation and he location of moving fronts is given as posteriori.

For one dimension, Stefan problem has been studied in depth, and there are many excellent numerical algorithms. However, the current algorithms have the limitations when those schemes are expanded to higher dimensions. One of the drawbacks is that those algorithms can not process with several frozen fronts, which may develop simultaneously and collapse each other. Recently another way to view moving interfaces was introduced by Osher and Sethian ${ }^{12}$. This level set method was first introduced to solve multiphase flow problems. The evolving interface on Stefan problem can be handled easily by the level set method because it is based on Eulerian approach with a fixed grid rather than Lagrangian approach such as front tracking method.

In the present work Stefan problem is formulated and solved using finite element method, which has been rarely used in tracking moving boundaries. Without applying indirect properties such as enthalpy or the freezing index, the energy equation is applied to an entire domain and the moving front is tracked by the level set method.

\section{Formulation of Stefan Problem}

Two-phase problem is considered in the present work. It is assumed that there is no mushy zone between solid and liquid phase and no induced inside motion by natural convection. Microscopic analysis such as crystal growth and dendritic solidification, which is specified by the classical Gibbs-Thomson relation, is not included. The governing equations for the Stefan problem are

$$
\begin{array}{ll}
\rho c_{s} \frac{\partial T}{\partial t}=\nabla \cdot\left(\mathbf{k}_{s} \nabla T\right) & \mathbf{x} \in \Omega^{s}{ }_{(\text {solid })} \\
\rho c_{l} \frac{\partial T}{\partial t}=\nabla \cdot\left(\mathbf{k}_{l} \nabla T\right) & \mathbf{x} \in \Omega^{l}{ }_{(\text {liquid })}
\end{array}
$$

where $\rho, c$ are density and specific heat capacity of each phase, and $\mathbf{k}$ is the thermal conductivity of material in $\Omega^{s}$ and $\Omega^{l}$. Initial condition and boundary conditions are as follows.

$$
\begin{aligned}
& \text { IC }: T(\mathbf{x}, t=0)=g(\mathbf{x}) \\
& B C S: 1 . \mathbf{x} \in \Gamma(\text { domain }) \\
& \text { Dirichlet or NeumannConditions } \\
& 2 . \mathbf{x} \in \Gamma(\text { interface }) \\
& T(x, t)=T_{m} \\
& \rho L V=-\left[\mathbf{k}_{l} \frac{\partial T}{\partial \mathbf{n}}-\mathbf{k}_{s} \frac{\partial T}{\partial \mathbf{n}}\right]
\end{aligned}
$$

On moving interface we have a jump condition (Stefan condition), which is formulated by energy balance at the interface. $L$ is the latent heat of melting/solidification and $T_{m}$ is either the melting or solidification temperature.

\section{Implicit Front Tracking by Level Set Method}

\section{A. Level Set Method}

The level set method ${ }^{13}$ is used to represent the evolution of moving interface by melting or solidification. There are two types of conventional approaches for tracking a moving front: One is Lagrangian and the other is Eulerian. For example, marker and particle method is Lagrangian approach and VOF (Volume of Fluid) is Eulerian approach. Both listed methods have their drawbacks. The former is the front tracking method and a serious problem can appear when several moving fronts expand and collapse. Originally it is developed to track arbitrary shape of moving boundary exactly but it is limited to one continuous boundary. The later is the fixed domain method and it can neither represent directional velocity fields accurately nor track the boundaries sharply. Level set method puts moving interface into one higher dimension. Then, the limitations of both methods can be overcome by tracking moving interface implicitly through an embedded higher dimensional domain. The equation of motion governing level set method is given by

$$
\phi_{t}+F|\nabla \phi|=0
$$


where $F$ is normal velocity at the front and $\phi$ is level set function. The position of the front is determined by the zero level set of a higher-dimensional function $\phi$. The level set function $\phi$ is defined initially as a signeddistance function such as

$$
\begin{aligned}
& \phi(\mathbf{x}, t=0)= \pm d(\mathbf{x}) \\
& \text { where } d(\mathbf{x})=\min _{\mathbf{y} \in \Gamma}\|\mathbf{x}-\mathbf{y}\|
\end{aligned}
$$

where $\Gamma$ is the set of moving fronts. To develop the weak form, for the finite element method, of the governing level set equation, normal velocity is changed such as

$$
\mathbf{u}=F \mathbf{n}=F \frac{\nabla \phi}{|\nabla \phi|}
$$

where $F$ is the magnitude of normal velocity and $\mathbf{n}$ is the unit normal vector of evolving interface. Then, we can solve the heat energy equation with advection, which is coupled to the above velocity, instead of solving the original level set governing equation. The governing equation to be solved for level set method is

$$
\left\{\begin{array}{l}
\phi_{t}+\mathbf{u} \cdot \nabla \phi=0 \\
\mathbf{u}=F \mathbf{n}=F \frac{\nabla \phi}{|\nabla \phi|}
\end{array}\right.
$$

It is the transient advection equation without any heat source or sink. To solve this type of governing equation, two stability schemes should be applied. Both spatial stabilization and temporal stabilization schemes are presented in the next section.

\section{B. Spatial Stabilization Scheme}

The advection heat problem, which is exactly the same as Eq. (6), has two problems when it is solved by numerical schemes. One is from the unsymmetric stiffness matrix and the other is instability of solution field. The latter one is relatively more significant problem. The wiggles will appear on the results or the solution will finally break down under convection dominated problem. The criteria for stability with a fixed mesh is defined by $P e$ number such as

$$
P e=\frac{\|\mathbf{u}\| \cdot h}{2 k} \leq 1
$$

However, this restriction has been overcome by several methods: artificial conductivity, SUPG (Streamline Upwind/Petrov-Galerkin), and GLS (Galerkin Least
Squares). In the present work SUPG is implemented to suppress the instability of numerical scheme ${ }^{14,15}$. The basic idea is to add diffusion (or viscosity) which acts only in the flow direction. Because the weighting function and interpolation functions are different in SUPG, the weighting function is defined as

$$
W_{I}=N_{I}+\frac{\alpha h}{2} \frac{u_{k}}{|\mathbf{u}|} \frac{\partial N_{I}}{\partial x_{k}}
$$

where $N_{I}$ is standard interpolation function in Galerkin method, $h$ is mesh size, $u_{k}$ is advection velocity and $\alpha$ is a weighting parameter for SUPG scheme. After assembling the mass and stiffness matrix by applying SUPG method, a system of simultaneous ordinary differential equations can be obtained as

$$
\mathbf{M} \dot{\Phi}+\mathbf{K} \Phi=\mathbf{0}
$$

where the mass and stiffness matrix are

$$
\begin{aligned}
& \mathbf{M}=\int\left(N_{I} N_{J}+\frac{\alpha h}{2} \frac{1}{|\mathbf{u}|} u_{k} \frac{\partial N_{I}}{\partial x_{k}} N_{J}\right) d \Omega \\
& \mathbf{K}=\int\left(N_{I} u_{p} \frac{\partial N_{J}}{\partial x_{p}}+\frac{\alpha h}{2} \frac{1}{|\mathbf{u}|} u_{k} \frac{\partial N_{I}}{\partial x_{k}} u_{p} \frac{\partial N_{J}}{\partial x_{p}}\right) d \Omega
\end{aligned}
$$

and $I, J=1, \ldots, N N O D E$ and $k, p=1, \ldots N D I M$.

\section{Temporal Stabilization Scheme}

For the temporal discretizaiton there are two methods: single-step and multi step schemes. It is well know that CFL condition should be satisfied for explicit schemes. This condition is defined as

$$
C F L=\frac{\|\mathbf{u}\| \cdot \Delta t}{h} \leq 1
$$

This criteria means the time increment $t$ should be restricted within a certain range with respect to the spatial increment to avoid instability. There are several methods for temporal discretization as $\theta$-method and Crank-Nicholson for a single step scheme, and Newton and Runge-Kutta method for multi steps scheme. In the present work, $\theta$-method is applied.

After applying $\theta$-method to Eq. (9), the following formulation is set up.

$$
\phi_{j}^{n+1}=(\mathbf{M}+\Delta \boldsymbol{\theta} \cdot \mathbf{K})^{-1}(\mathbf{M}-\Delta t(1-\theta) \cdot \mathbf{K}) \phi_{j}^{n}
$$

where $\theta=0$ explicit Euler forward, $\theta=1 / 2$ CrankNicholson, $\theta=2 / 3$ Galerkin, $\theta=1$ implicit Euler 
backward method. Crank-Nicholson method gives us the most exact solution but it may produce some oscillation in the solution field. The implicit scheme is unconditionally stable but the solution may diffuse too much and leave an error. To solve the Eq. (12), iteration method is applied. In the present work, DSDBCG (diagonal scaled bi-conjugate gradient method) from SLATEC library is implemented to solve the time/space discretized equations.

\section{Numerical Formulation}

The transient heat diffusion equation is presented in the weak form, which is the setting for the finite element implementation of the equation. The mathematical formulation is given on section II. In the transient heat diffusion problem, the solution possesses somewhat the 'best approximation' property ${ }^{14}$. That is, the difference between the finite element solution and the exact solution is minimized with respect to a certain norm. The success of standard Galerkin method is largely due to the 'best approximation' result. Then, the Stefan problem here is formulated by Galerkin method without any involvement of the spatial stability scheme. However, there is a difficulty to represent the problem variationally under the whole domain because the location of moving interfaces is coupled to the governing equation. Without this information we cannot specify necessary boundary conditions on the moving fronts. This difficulty can be overcome by implementing level set method to track the moving front effectively before attempting to solve the diffusion equation.

Consider a whole domain $\quad 3$ of which is occupied by two phases materials. Liquid phase occupies the domain ${ }^{l}$ and solid phase occupies the domain ${ }^{s}$. At the interface, , which is moving in the direction of its normal $\mathbf{n}$ with speed $F$, the Stefan condition is specified. The weak form of the energy equation throughout the region is given as

$$
\begin{gathered}
\left(w, \rho c T_{, t}\right)_{\bar{\Omega}}+(\nabla w, \mathbf{k} \nabla T)_{\bar{\Omega}}=(w, q)_{\partial \bar{\Omega}}+(w, h T)_{\partial \bar{\Omega}} \\
\text { where } \quad \mathbf{x} \in \bar{\Omega}=\Omega^{l} \cup \Omega^{s}
\end{gathered}
$$

The space of weighting functions, $W$, and the admissible space of trial functions, $K$, given by

$$
\begin{gathered}
W=\left\{w: w \in H^{1}(\Omega) \mid w=0 \text { on } \partial \Omega\right\} \\
K=\left\{T: T \in H^{1}(\bar{\Omega}) \mid T=g \text { on } \partial \Omega\right\}
\end{gathered}
$$

where $\Omega=\Omega^{1} \cup \Omega^{\mathrm{s}} \cup \Gamma$. The weak form (13) is solved by finally applying the necessary conditions on the moving interfaces by a penalty method after tracking the fronts implicitly by level set method. $\theta$-method is implemented to the weak form and the discretized finite element equations are shown on Eq. (16) and (17). Biconjugate gradient iteration method is applied to solve the set of ordinary differential equations.

$$
\phi_{j}^{n+1}=(\mathbf{M}+\Delta \theta \cdot \mathbf{K})^{-1}(\mathbf{M}-\Delta t(1-\theta) \cdot \mathbf{K}) \phi_{j}^{n}
$$

where the mass and stiffness matrix is given as

$$
\mathbf{M}=\int\left(N_{I} N_{J}\right) d \Omega \quad \mathbf{K}=\int\left(\frac{\partial N_{I}}{\partial x_{i}} k_{i j} \frac{\partial N_{J}}{\partial x_{j}}\right) d \Omega
$$

\section{$\underline{\text { V. Reinitialization and Extension Velocity }}$}

\section{$\underline{\text { A. Reinitialization }}$}

Reinitialization is a preferable method to avoid having steep or flat gradients develop in level set function $\phi$. The level set function has to keep the exact signed distance from the evolving interfaces. In fluid flow problems, which directly use a fluid velocity calculated on the entire domain, the level set function might distort or stretch the function, $\phi$. Without this procedure the moving front may stop evolving anymore and violate the conservation of mass ${ }^{16}$. There are several ways to accomplish the reinitialization. Sussman et $a l^{16}$ applied 'sign' function for reinitialization by solving the specially devised partial differential equation. Chopp's ${ }^{17}$ minimum surfaces method simply recalculated the signed distance function by standing at each grid point. Adalsteinsson and Sethian ${ }^{18}$ implemented Fast Marching Methods, which is used to solve the Eikonal equation, to reinitialize the level set function. In the present work the sign-method which was introduced by Sussman et $a l^{16}$ is implemented. Its advantage comparing with other methods is that the reinitialization can be done without explicitly finding the zero level set.

The obtained level set function $\phi_{o}(\mathbf{x})$ at each time step need not be a distance function. However, its zero level set is the contour of the moving interfaces. Then, we can construct the exact signed distance function $\phi(\mathbf{x})$, which should have the same zero level set to $\phi_{o}(\mathbf{x})$, by solving the following equation until it reaches steady state

$$
\begin{aligned}
& \phi_{t}=S\left(\phi_{0}\right)(1-|\nabla \phi|) \\
& \text { where } \quad S\left(\phi_{0}\right)=\frac{\phi_{0}}{\sqrt{\phi^{2}+\varepsilon^{2}}}
\end{aligned}
$$

By letting $\mathbf{a}=S\left(\phi_{o}\right) \mathbf{n}$ and $f=S\left(\phi_{o}\right)$, the variational formulation of the Eq. (18) can be developed from Eq. (19). 


$$
\begin{aligned}
& \phi_{t}+\mathbf{a} \cdot \nabla \phi=f \\
& \text { where } \mathbf{a}=S\left(\phi_{o}\right) \mathbf{n}=S\left(\phi_{o}\right) \frac{\nabla \phi}{|\nabla \phi|}, \quad f=S\left(\phi_{o}\right)
\end{aligned}
$$

The finite element formulation of Eq. (19) is almost same to that of the level set equation at section III, and then we avoid the reproduction of formulation here. Sussman et ll $^{16}$ solved Eq. (18) by finite difference method, then both $\phi(\mathbf{x})$ and $S\left(\phi_{o}\right)$ are grid functions. However, in the FE formulation for Eq. (19), a is element-based function and $S\left(\phi_{o}(\mathbf{x})\right)$ is node-based function. The transition of the signed function from node-based to element based function is done by considering values of all nodes in one element. For example, consider the following four node linear QUAD elements. Three basic cases are shown on Fig. 1 and defined as Eq. (20). By constructing sign function of each element from Eq. (19), the reinitialization equation is solved by finite element method

$$
\begin{aligned}
& \sum_{I=1}^{\text {nnode }} \phi_{I}=\phi_{1}+\phi_{2}+\phi_{3}+\phi_{4} \\
& = \begin{cases}<0, & S\left(\phi_{0}\right)=-1 \\
=0, & \text { if one of } \phi_{I}=\left(\begin{array}{l}
<0, S\left(\phi_{0}\right)=-1 \\
>0, S\left(\phi_{0}\right)=+1
\end{array}\right. \\
>0, & S\left(\phi_{0}\right)=+1\end{cases}
\end{aligned}
$$

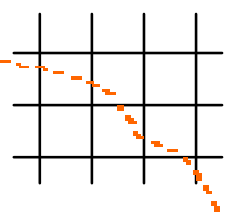

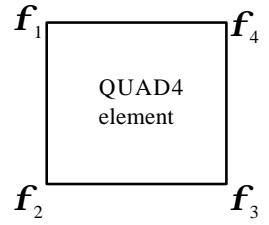

Fig. 1 Three different cases of sign function and QUAD 4 linear element

\section{B. Front Tracking and Extension Velocity}

One of the appealing features of level set methods is that the front need not be explicitly constructed and that all of the methodology may be executed on the imbedded domain. In the present work we try to adhere to this advantage so that the sign method is adopted at the reinitialization step before. Even if the evolving front can be traced implicitly, the locations of nodes neighboring the front have to be detected. There are two major reasons to be done. One is to determine the normal velocity of the moving fronts by the jump condition (Stefan condition) at the front. Second is to set the Dirichlet boundary conditions on the moving front to solve the energy governing equation. The simple method to detect the front is devised here. The moving locations are remembered through a specially arranged array in this way. The recommended size of $\varepsilon$ is $h$ or $0.5^{*} h$ ( $h$ is mesh size).

Somewhat behind facts about the speed function $F$ on the front comes from the level set Eq. (3). The speed $F$ should be defined through the entire embedded domain. $F$ may include two different velocities in the fluid/thermal problems: passive advection velocity and local velocity of the specific location of the interface, which is set by the jump condition. In the Stefan problem, the speed function is given only by the jump condition (Stefan condition) at the moving interface. Then, this speed function should be defined all over the embedded domain to solve the level set equation.

Sethian and Strain ${ }^{19}$ solved crystal growth and dendritic solidification by combining a boundary integral method with a level set method. This is for constructing the normal velocity on the front and extending this velocity to the other embedded domain to construct an extension velocity. Chen et $a l^{20}$ developed an extension velocity by solving an advection equation in each component directly to avoid a complicated incorporation with boundary integral method. However, there is considerable room to maneuver an extension velocity. The only constraint for the extension velocity is

$$
\begin{aligned}
& \lim _{\mathbf{x} \rightarrow \mathbf{a}} F_{\text {ext }}=F(\mathbf{a}) \\
& \text { where } \quad \mathbf{x}=\mathbf{a} \text { is location of moving front }
\end{aligned}
$$

In the present work, after detecting the front location, the normal velocity at the moving front is constructed by the jump condition and this velocity simply extended to off the front.

\section{Outline of Algorithm}

The steps to solve Stefan problem by level set method are outlined as follows.

1. Construct initial extension velocity

1-a Calculate initial distance function from the initial starting point

1-b Construct unit normal vectors at the front

1-c Build normal velocity at the front by the jump condition (Stefan condition)

1-d Construct extension velocity after detecting moving fronts

2. Solve level set equation

2-a Assembly for finite element global mass and stiffness matrix

2-b Assembly for $\theta$-method (transient)

2-c Solve it by DSDBCG iteration scheme

3. Reinitialization of distance function

3-a Build sign function

3-b Assembly for finite element global mass and stiffness matrix

3-c Assembly for $\theta$-method (transient) 
3-d Solve it by DSDBCG iteration scheme 3-e Repeat 'a-d' until it reaches steady state 3-f Steady state should reach within several iterations

4. Solve Stefan problem (melting/solidification)

4-a Detecting updated moving fronts

4-b Assembly for finite element global mass and stiffness matrix and apply necessary boundary conditions on the updated moving fronts

4-c Assembly for $\theta$-method (transient)

4-d Solve it by DSDBCG iteration scheme

5. Construct extension velocity

5-a Calculate the first derivative of temperature

5-b Construct unit normal vectors at the front

5-c Build normal velocity at the front by the jump condition (Stefan condition)

5-d Construct extension velocity

Repeat 1-4 for each time step

\section{Numerical Examples}

A. Single Phase One Dimensional Solidification

In one dimension, Stefan problem has been studied in depth, and there are many excellent numerical algorithms for solving them. The exact similarity solution is obtained as ${ }^{2,3}$

$$
u(x, t)=U_{0}-\frac{U_{0}}{\operatorname{erf}(\alpha / 2 \sqrt{k})} \operatorname{erf} \frac{x}{2 \sqrt{k t}}
$$

where $u(0, t)=U_{0}$, and $\alpha$ is determined by solving the following equation.

$$
\lambda e^{\lambda^{2}} \operatorname{erf}(\lambda)=\frac{U_{0} c}{L \sqrt{\pi}} \quad \text { where } \lambda=\frac{\alpha}{2 \sqrt{k}}
$$

For the problem $\alpha=1.24013$ and the results from two solutions are given at Fig. 2.

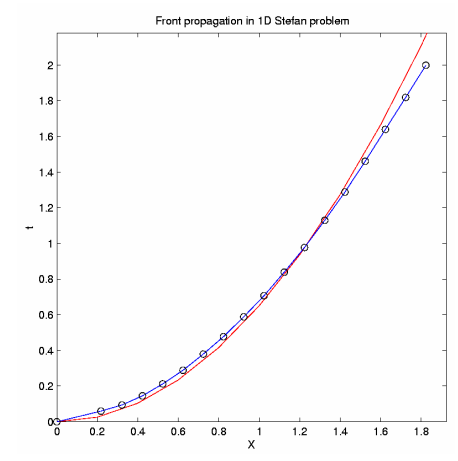

Fig. 2 Comparison with the exact similarity solution for single-phase and 1D solidification

\section{B. Two Phase Two Dimensional Solidifications}

B.1 Two Starting Points on the Boundary This example is selected from the work of Yichikawa and Kikuchi ${ }^{11}$ to see the collapse of moving fronts and its topology change. Total $1600=40 \times 40$ elements are used for the finite element modeling and its real dimension is $1 \times 1 \mathrm{~cm}^{2}$, which is centered at $(0,0)$. For boundary conditions, relative high temperature $\left(T_{\text {high }}=1\right.$ $\left.{ }^{\circ} C\right)$ is set on two points on the boundary: $(-0.5,-0.1)$ and $(-0.1,-0.5)$, which initiate melting of the domain. Homogeneous Neumann boundary condition is applied all other surround edges of the domain. Initial temperature of the domain is $T(x, y, t=0)=-1{ }^{\circ} C$, which represents solid state at the beginning of analysis. Density and thermal heat capacity are set to unity and isotropic thermal conductivity is assumed. Time step $(d t)$ of the simulation is set to 2.E-3 and the entire transient analysis is done until $t=0.05 \mathrm{~s}$. As shown on Fig. 3, the topological change as two moving melting fronts collapse can be handled naturally by the level set method.

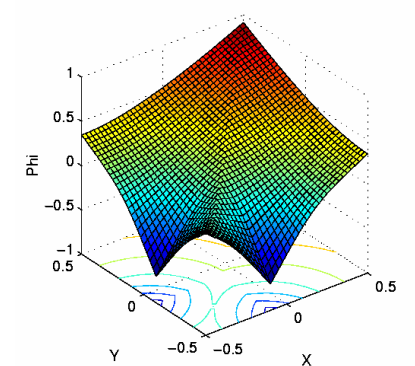

(a)

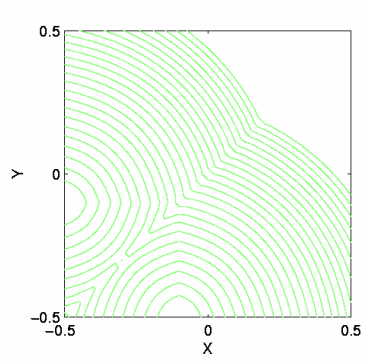

(b)
Fig. 3 (a) Level set surface and its contour at $t=0.014 \mathrm{~s}$; zero level set is the moving front (b) History of moving melting fronts at each time step which come from the zero level set of level set function at each time step $(d t=2$.E-4)

\section{B. 2 Two Starting Points inside the Domain}

Next example for two-dimensional Stefan problem is considering the starting of melting from the inside of the domain. Relative high temperature $\left(T_{\text {high }}=1{ }^{\circ} C\right)$ is set on two points on the domain inside: $(-0.25,0.25)$ and $(0.25,-0.25)$. There is no boundary heat convection from the surroundings. Initial temperature of the domain is $T(x, y, t=0)=-1{ }^{\circ} C$, which represents solid state at the beginning of analysis. Finite element model and its real dimension are exactly same as the example B.1. Density and thermal heat capacity are set to unity and isotropic thermal conductivity is assumed to be unity. Time step $(d t)$ of the simulation is set to $1 . \mathrm{E}-3 \mathrm{~s}$ and the entire transient analysis is done until $t=0.025 \mathrm{~s}$. The result of tracking the melting fronts is shown on Fig. 4.

\section{Two Phase Three Dimensional Solidification}




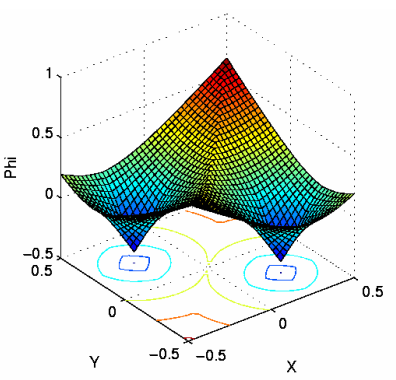

(a)

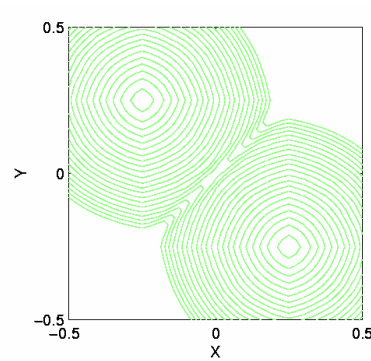

(b)
Fig. 4 (a) Level set surface and its contour at $t=0.07$ sec. (b) History of moving melting fronts at each time step which come from the zero level set of level set function at each time step $(d t=1$.E-3)

Stefan problem of three-dimensional cases is investigated. By the level set method, the analysis of moving front problem can be handled quiet easily by extending the ideas to higher dimension. The visualization of level surface in three dimension is somewhat unusual but the level set function should represent the exact distance from the moving surfaces. After the construction of the exact initial distance function, the distance can be kept accurately by reinitialization at every time step. Two illustrative examples are given with and without boundary heat convection.

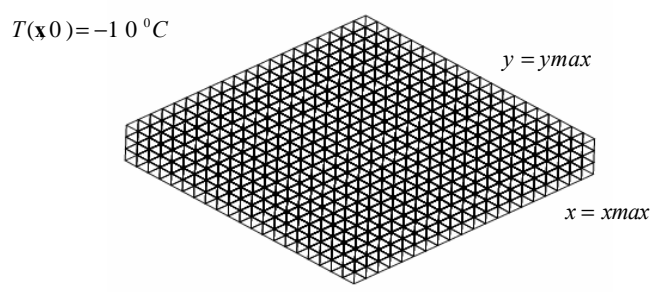

Fig. $520 \times 20 \times 3$ finite element model for three dimensional Stefan problem

\section{C.1 Four Starting Edges}

Total $1200=20 \times 20 \times 3$ elements are constructed for three dimensional finite element model. The real dimension of the model is $1 \times 1 \times 0.3 \mathrm{~cm}^{3}$, which is centered at $(0,0 ., 0$.$) . For boundary conditions, relative high$ temperature $\left(T_{\text {high }}=1{ }^{\circ} \mathrm{C}\right)$ is set on four corner edges, from which melting starts. Initial temperature of the domain is $T(x, y, t=0)=-1{ }^{\circ} C$. Time step $(d t)$ of this simulation is set to $1 . E-3$ and the entire transient analysis is done until $t=0.1 \mathrm{~s}$. Isotropic thermal conductivity $\mathbf{k}=1.0 \mathrm{~W} / \mathrm{mK}$ is applied. Density and thermal heat capacity is set to unity. Transient results at various timestep are shown on Fig. 6.
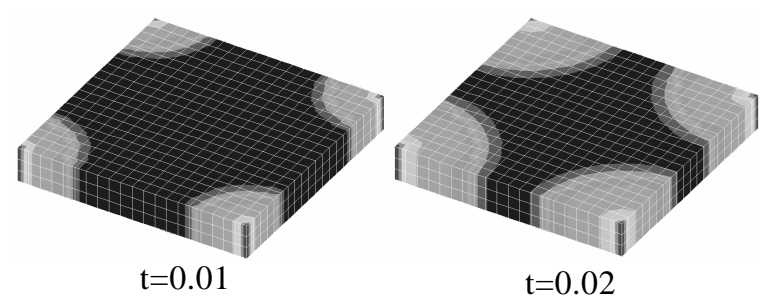

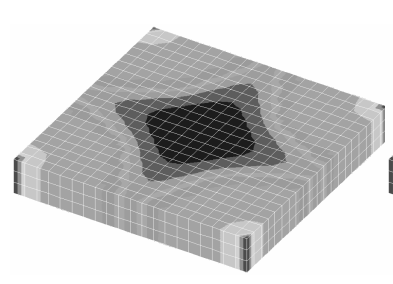

$\mathrm{t}=0.035$

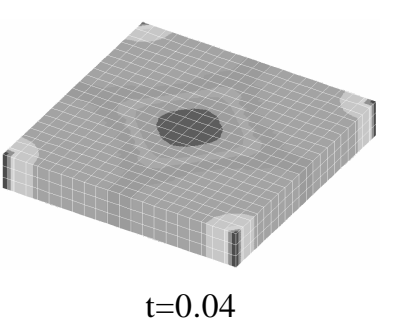

at $t=0.01,0.02$, 0.035 and $0.04 \mathrm{~s}$. Melting starts from the four corner edges and the growth and collapse of melting fronts are shown. Time step $d t$ is set to 1.E-3 and the time dependent simulation is done until it reaches $0.1 \mathrm{~s}$.

\section{C.2 One Starting Edge with Boundary Heat}

\section{Convection}

Boundary heat convection phenomena is included. The exact same finite element model $(20 \times 20 \times 3)$ is constructed as shown on Fig. 5. However, real dimension of the model here is $2 \times 2 \times 0.3 \mathrm{~cm}^{3}$. From the Fig. 5, $x=x \max$ and $y=y \max$ surfaces are surrounded by relative high temperature fluid. The heat convection coefficient $h_{\infty}$ is $1 \mathrm{W/m} \mathrm{m}^{2} \mathrm{~K}$ and surrounding fluid temperature $T_{\infty}$ is $1{ }^{\circ} \mathrm{C}$. All other surfaces are insulted. One corner edge on the left bottom side is specified Dirchlet boundary condition as $T=T_{\text {high }}=10{ }^{\circ} \mathrm{C}$. The initial solid state temperature is set to $T(x, y, z, t=0)=-$ $10^{\circ} \mathrm{C}$, which is shown as dark black color on the Fig. 7 . Density and thermal heat capacity is assumed to be unity and isotropic thermal conductivity is set to $\mathbf{k}=100$ WImK.

\section{Conclusion}

Stefan problem has been studied extensively until so far by two major categories. One is the front tracking method and the other is the fixed domain method. All the major computations have been done by finite difference method and the finite element method is seldom used before. Variational formulation using finite element formulation can be applied only to the fixed domain method involving the indirect properties because it is difficult to handle the jump condition at the moving interfaces. This drawback can be overcome by combining the level set method, which puts the evolving interface into an imbedded domain (one higher dimension), with the finite element formulation. From the result of the present work, the combined 
algorithm can be extended to higher dimensional problem.
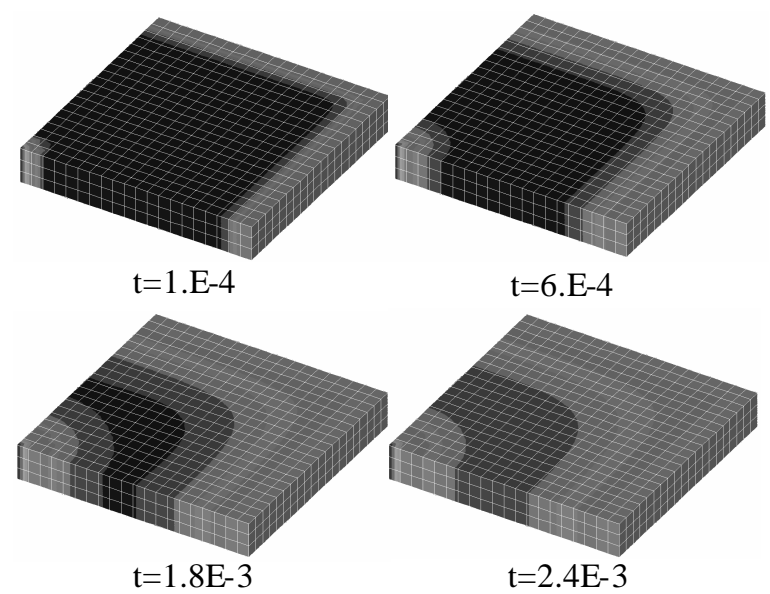

Fig. 7 Temperature distributions at various time steps. Melting starts from both one left-bottom corner edge and two surrounding surfaces. Time step $d t$ is set to $1 . \mathrm{E}-4$ and the time dependent simulation is done until it reaches $2.5 \mathrm{E}-3 \mathrm{~s}$.

\section{$\underline{\text { References }}$}

${ }^{1}$ Carslaw, H.S. and Jaeger, J.C., Conduction of Heat in Solids, Clarendon Press, Oxford 1959, Chapter 11

${ }^{2}$ Tikhonov, A.N., Equations of Mathematical Physics, Pergamon Press, Oxford 1963

${ }^{3}$ Crank, J., Free and moving boundary problems, Clarendon press, Oxford 1984

${ }^{4}$ Crank, J., "How to Deal with Moving Boundaries in Thermal Problems," Numerical Methods in Heat Transfer edited by R.W.Lewis, K.Morgan, and O.C.Zienkiewicz, John Wiley \& Sons Ltd, Chapter 9, 1981, pp 177-200

${ }^{5}$ Ehrlich, L.W., "A numerical method of solving a heat flow problem with moving boundary," Journal of the Association for Computing Machinery, Vol. 5, 1958, pp 161-176

${ }^{6}$ Koh, J.C.Y., Price, J.F., and Colony, R., 'On a Heat and Mass Transfer Problem with Two Moving Boundaries," Progress in Heat and Mass Transfer, Vol. 2, 1969, pp225-247

${ }^{7}$ Crank, J., "Two Methods for the Numerical Solution of Moving-Boundary Problems in Diffusion and Heat Flow', Quarterly Journal of Mechanics and Applied Mathematics, Vol. 10, 1957, pp220-231

${ }^{8}$ Lotkin, M., "Calculation of Heat Flow in Melting Solids," Quarterly of Applied Mathematics, Vol. 18, 1960, pp79-85

${ }^{9}$ Kim, C.J. and Kaviany, M., "Numerical Method for Phase-Change Problems," International Journal of Heat and Mass Transfer, Vol. 33, No. 12, 1990, pp2721-2734
${ }^{10}$ Fremond, M., "Variational Formulation of the Stefan Problem-Coupled Stefan Problem-Frost Propagation in Porous Media," International Conference on Computational Methods in Nonlinear Mechanics (University of Texas, Austin), 1974, pp341349

${ }^{11}$ Ichikawa, Y. and Kikuchi, N., "One-Phase MultiDimensional Stefan Problem by The Method of Variational Inequalities," International Journal for Numerical Methods in Engineering, Vol. 14, 1979; pp1197-1220

${ }^{12}$ Osher, S. and Sethian, J.A., "Fronts propagating with curvature-dependent speed: Algorithms Based on Hamilton-Jacobi formulations," Journal of Computational Physics, Vol. 79, No. 1, 1988; pp12-49

${ }^{13}$ Sethian, J.A., Level set methods and fast marching methods, Cambridge University Press 1999

${ }^{14}$ Brooks, A.N. and Hughes, T.J.R., "Streamline upwind/Petrov-Galerkin Formulations for Convection Dominated Flow with Particular Emphasis on the Incompressible Navier-Stokes Equations," Computer Methods in Applied Mechanics and Engineering, Vol. 32, 1982, pp199-259

${ }^{15}$ Kelly, D.W., Nakazawa, S., Zienkiewicz, O.C. and Heinrich, J.C., "Note on Upwinding and Anisotropic Balancing Dissipation in Finite Element Approximations to Convective Diffusion Problems," International Journal for Numerical Methods in Engineering, Vol. 15, 1980, pp1705-1711

${ }^{16}$ Sussman, M., Smereka, P., and Osher, S., "Level Set Approach for Computing Solutions to Incompressible Two-phase Flow," Journal of Computational Physics, Vol. 114, 1994, pp146-159

${ }^{17}$ Chop, D.L., 'Computing Minimal Surfaces via Level Set Curvature Flow," Journal of Computational Physics, Vol. 106, 1993; pp 77-91

${ }^{18}$ Adalsteinsson, D. and Sethian, J.A., "The Fast Construction of Extension Velocities in Level Set Methods," Journal of Computational Physics, Vol. 148, 1999; pp2-22

${ }^{19}$ Sethian, J. A. and Strain, J., "Crystal Growth and Dendritic Solidification," Journal of Computational Physics, Vol. 98, 1992, pp231-253

${ }^{20}$ Chen, S., Merriman, B., Osher, S., and Smereka, P., "Simple Level Set Method for Solving Stefan Problems," Journal of Computational Physics, Vol. 135, 1997; pp8-29 